\title{
Diseño de una estrategia de control para cargas electrónicas programables en microrredes
}

\author{
León F. Serna-Montoya, Juan B. Cano-Quintero, Nicolás Muñoz-Galeano y Jesús M. López-Lezama \\ Facultad de Ingeniería, Dpto. de Ingeniería Eléctrica, Universidad de Antioquia, Grupo de investigación GIMEL, \\ Medellín-Colombia (correo-e: leon.serna@udea.edu.co; bernardo.cano@udea.edu.co; nicolas.munoz@udea.edu.co; \\ jmaria.lopez@udea.edu.co)
}

Recibido Oct. 29, 2020; Aceptado Dic. 29, 2020; Versión final Ene. 18, 2021, Publicado Abr. 2021

\section{Resumen}

Este estudio presenta el diseño de una estrategia de control para cargas electrónicas programables (PEL, en inglés) en corriente alterna (AC, en inglés), aplicado a escenarios de pruebas en microrredes de energía. La estrategia de control se basa en control de corriente por histéresis (HCC, en inglés) para la operación de un inversor trifásico con el objetivo de emular perfiles de carga. El diseño de la PEL es dividido en etapas de control y potencia para identificar los principios operativos. La topología empleada es una PEL trifásica en AC con conexión en estrella de cuatro conductores y conexión del bus DC partido. La PEL es diseñada para operar en los modos de potencia, impedancia y corriente constante. Se concluye que la simulación de la PEL funciona de manera adecuada, representando las formas de onda de la etapa de potencia y las señales de control de acuerdo con la teoría.

Palabras clave: electrónica de potencia; carga electrónica programable; control de histéresis; microrredes; simulación

\section{Design of a control strategy for programmable electronic loads in microgrids}

\begin{abstract}
This study presents the design of a control strategy for alternating current (AC) programmable electronic loads (PEL) applied to test scenarios in power microgrids. The control strategy is based on hysteresis current control (HCC) for the operation of a three-phase inverter to emulate load profiles. The PEL's design is divided into power and control stages to identify operating principles. The topology used is a three-phase four-wire AC PEL, using star and split DC bus connections. The DC link voltage is controlled through a Buck converter based on the power balance of the system. The PEL has been designed to operate in constant power, impedance, and current modes. It is concluded that PEL simulations function properly, representing power stage wavelengths and control signs according to theory.
\end{abstract}




\section{INTRODUCCIÓN}

En los últimos años la industria eléctrica ha experimentado cambios de paradigmas motivados por avances tecnológicos que han dado viabilidad económica a la generación distribuida (GD) y una renovada conciencia ecológica. En este contexto, las microrredes (MRs) han tomado el rol de elementos gestores para la integración de GD en las redes de distribución (Arias et al., 2017; Gómez et al., 2018; Restrepo-Garcés et al., 2016). La masificación de MRs en redes de distribución ha traído nuevos desafíos a los ingenieros en términos de su apropiada gestión (Vukojevic et al., 2020; Manbachi et al., 2020; Saldarriaga-Zuluaga et al., 2020). Por esta razón, resulta conveniente realizar pruebas de funcionamiento a las MRs previo a su puesta en servicio. Dichas pruebas deben adecuarse a la flexibilidad, robustez y agilidad para el correcto dimensionamiento, diseño y operación de dispositivos eléctricos. Las pruebas a MRs se pueden realizar mediante el uso de PELs (programmable electronic loads).

En términos generales, las PELs son dispositivos de electrónica de potencia, encargados de generar perfiles de carga (LPs, Load Profiles) para evaluar la respuesta de los equipos bajo prueba (Geng et al., 2018). Estos equipos incluyen fuentes de potencia ininterrumpidas, cargadores de baterías, inversores de sistemas fotovoltaicos, inversores eólicos y sistemas de carga de vehículos eléctricos. En la literatura científica se han reportado gran variedad de topologías de hardware usadas en la aplicación de PELs. En Novak et al., (2015) se presentan aplicaciones de PELs que usan el convertidor Buck en el enlace DC para disipar los excesos de potencia. En contraposición, las PELs diseñadas con retroalimentación de energía a la red solo disipan una cantidad mínima de energía que corresponde a las pérdidas de conmutación y conducción. Este tipo de PEL también es llamado emulador de red ya que permite la operación en los cuatro cuadrantes; es decir, funcionan como fuente y como sumidero (Aurilio et al., 2013; Geng et al., 2018). En Geng et al., (2018) se presentan PELs con una topología de inversor multinivel, permitiendo una emulación con menor contenido armónico. Este artículo propone el uso de la topología reportada en Novak et al., (2015) debido al enfoque de operación como carga; es decir, sólo la operación de los cuadrantes de consumo de energía. Esta topología consiste en un inversor de tres ramas con conexión a una fuente trifásica de conexión en estrella y el punto de referencia o neutro se toma del punto medio de dos capacitores que conforman el bus DC, generando una referencia simétrica. El convertidor Buck es empleado en el bus DC para disipar los excesos de potencia y mantener la tensión estable (Kim et al., 2019).

La principal contribución de este artículo es el desarrollo de una estrategia de control para PELs basada en HCC para el control de corriente en el lado AC y basado en control PI para el control del bus DC, mostrando el detalle de la operación del sistema y la gestión de la energía. El HCC considera una banda de referencia y por medio del accionamiento de los interruptores de potencia incrementa o decrementa el nivel de corriente percibido por el equipo bajo prueba (Kapat, 2017). La estrategia de control con HCC en el lazo interno más control PI en el lazo externo es la más común en la literatura científica.

En Aurilio et al., (2013) se describen controladores PI en cascada; por otro lado, en Novak et al., (2015) se describen los controladores PI considerando la trasformación de Park, mientras en Geng et al., (2018) se presentan los mismos con variables de estado. En Srinivasa y Chandorkar, (2010) se usa un controlador de retroalimentación óptimo denominado regulador cuadrático lineal. Esta técnica permite ponderar el peso de las variables de estado facilitando la sintonización de los lazos de control.

Este artículo también describe detalladamente el principio de operación de las PELs, analizando los resultados mediante simulaciones. El esquema de potencia corresponde a un inversor de tres ramas, con bus DC partido y resistores de estabilización; además, se utiliza un convertidor Buck en el enlace DC para disipar los excesos de energía. Los esquemas de control HCC del inversor (VSI) y del convertidor Buck son analizados, simulados y validados, comprobando la estabilidad y funcionamiento de la PEL. Para validar la operación; los modos de operación de potencia, impedancia y corriente constantes fueron simulados y sometidos a perturbaciones de tensión, evidenciando una respuesta apropiada de la PEL.

\section{MATERIALES Y MÉTODOS}

La Figura 1 describe la topología de la PEL y su esquema de control. La topología consiste en un VSI (Voltage Source Inverter) de tres ramas con bus DC partido (C1 y C2) y un convertidor Buck que permite la disipación del exceso de energía en el bus DC, regulando su tensión. La PEL es la carga de las fuentes de tensión alterna y genera los perfiles de carga necesarios para los equipos bajo prueba. Los esquemas de control son: 1) el control HCC que permite controlar la corriente en el lado de AC del inversor y 2) el control del convertidor Buck basado en un controlador PI. El control HCC requiere un lazo de seguimiento de fase (PLL, Phase-Locked Loop) y un generador de referencias para las corrientes. Las señales de los sensores son ilustradas por líneas discontinuas, relacionando las señales de voltaje y corriente. Las señales de control de los interruptores son ilustradas en líneas punteadas. El inversor se representa mediante el uso de tres recuadros que representan sus tres ramas; también, el control HCC y el generador de referencias aparecen representados cada uno con 
tres recuadros dado que requieren el procesamiento de tres señales. El convertidor Buck es un sistema de un único circuito, por ende, el Buck y su controlador se representan cada uno mediante un único recuadro. El PLL se utiliza para obtener el ángulo de la tensión de la fase a; por lo tanto, se representa mediante un solo recuadro.

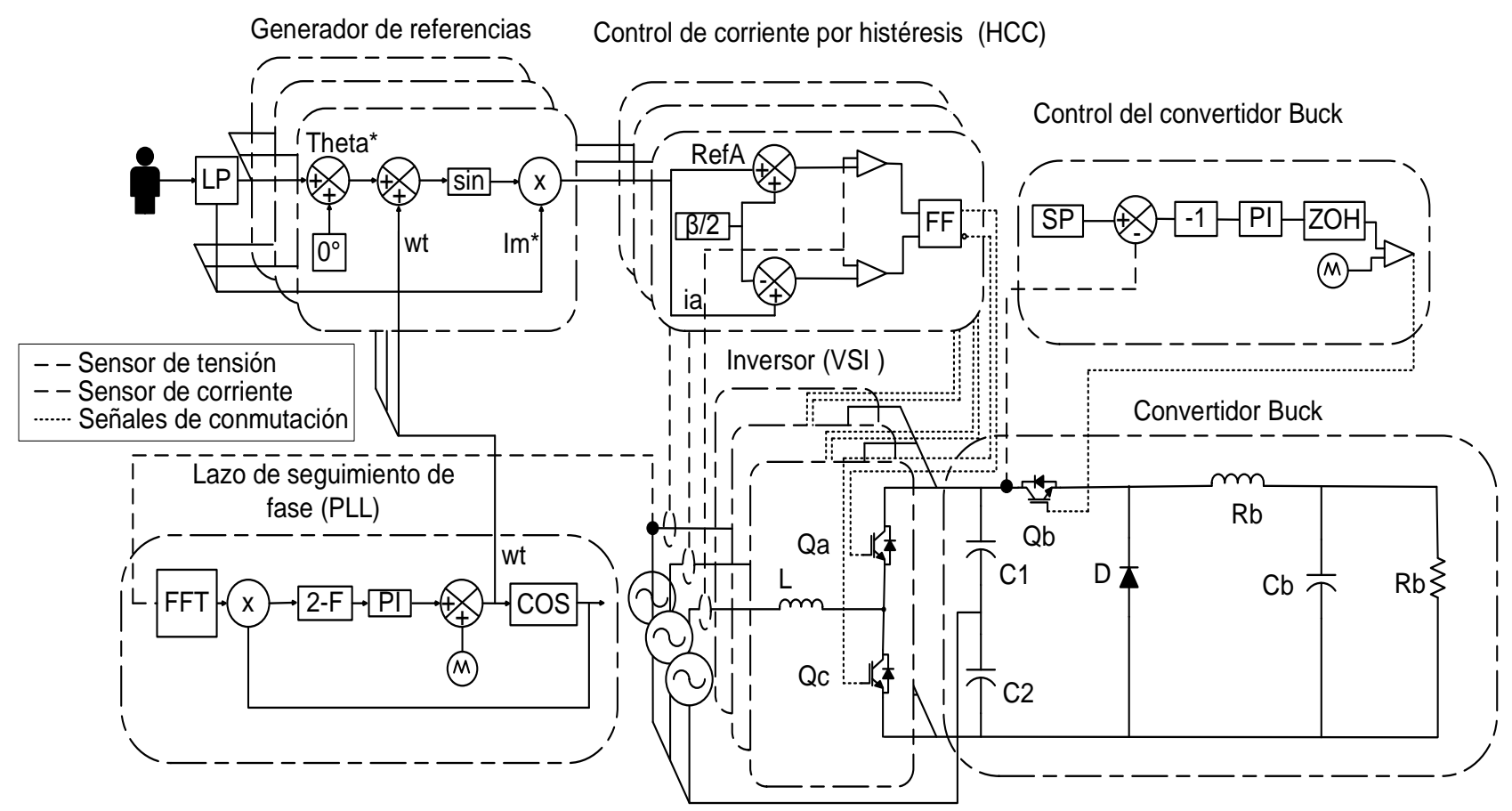

Fig. 1: Diagrama general de control y potencia de una PEL

El sistema "Generador de referencias" tiene como entrada el bloque LP en el cual el usuario debe ingresar el perfil de carga deseado que corresponde a las corrientes de referencia para la PEL; en su salida, el bloque LP entrega la magnitud de la corriente deseada $\left(\mathrm{Im}^{*}\right)$ y también el ángulo (Theta*), que es el desfase respecto a cada ángulo del sistema trifásico $\left(0^{\circ},-120^{\circ}, 120^{\circ}\right)$. El sistema "Generador de referencias" requiere la sincronización con la red de la fuente alterna; por eso toma de la salida del sistema PLL la frecuencia angular multiplicada por el tiempo del sistema ( $\omega t)$. El sistema PLL requiere de la medida de la forma de onda del voltaje de la fase a, luego este voltaje es procesado mediante el bloque FFT que realiza la transformada rápida de Fourier (FFT, Fast Fourier Transform) y obtiene la magnitud y el ángulo del armónico fundamental del voltaje. Las referencias generadas se desfasan 120 grados entre sí para poder emular sistemas trifásicos balanceados. Con base en las referencias y la medición de las corrientes (ia, ib, ic) que ingresan a la PEL, la etapa "Control de corriente por Histéresis (HCC)" es la encargada de accionar los interruptores de potencia (Qa y Qc) del inversor VSI para generar los LPS.

La energía que ingresa al VSI se gestiona como sigue: 1) Si el LP corresponde a un perfil de potencia activa (flujo unidirecional), el bus DC debe disipar la energía; para esto, utiliza el convertidor Buck que se encarga de compensar los excesos de potencia del bus $\mathrm{DC}$, mediante la disipación térmica en el resistor (Rb), a través de la conmutación del interruptor (Qb) (Bloque Control del Convertidor Buck). 2) Si el LP corresponde a un perfil de potencia reactiva (flujo bidireccional), el VSI gestiona la entrada y salida de la energía produciendo una potencia media igual a cero; es decir, la potencia reactiva no aumenta el nivel del bus DC pero si influye en su rizado. A continuación, se explican en detalle cada uno de los bloques enunciados previamente.

\section{Generación de referencias}

Los tipos de cargas más comunes reportadas en Aurilio et al., (2013) son: corriente, potencia e impedancia constantes. Estos son mostrados respectivamente en las Figuras 2 a, b y c. La ecuación (1) describe la función matemática para generar una referencia, construyendo un perfil de corriente constante. Note que los parámetros de magnitud $\left(\mathrm{Im}^{*}\right)$ y ángulo de desfase (Theta*) son definidos por el usuario. El argumento ( $\left.\omega t\right)$ es estimado por el PLL, determinando la referencia de la señal. La etapa denominada "Generación de referencias" tiene asociado un ángulo para formar un desfase de $120^{\circ}$ entre ellos; por ende, el recuadro superior de la Figura 1 ilustra un ángulo de cero grados, los dos rectángulos interiores tienen ángulo de $-120^{\circ}$ y $120^{\circ}$ para originar las referencias de un sistema trifásico positivo y balanceado.

$i(t)=I_{m}^{*} \sin \left(\omega t+\theta^{*}+0^{\circ}\right)$ 


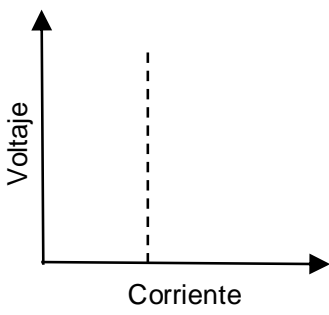

a)

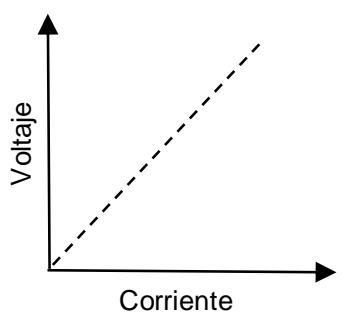

b)

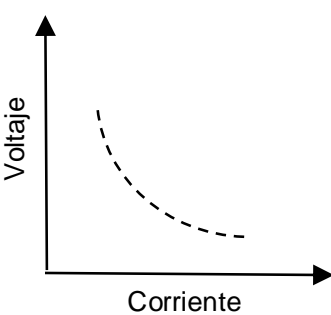

c)

Fig. 2: Perfiles de carga constantes

EI LP de potencia constante se puede expresar como un LP de corriente en función de la tensión de la red de alimentación, determinando la magnitud del voltaje con la transformada rápida de Fourier (FFT) y el ángulo deseado con base en la referencia ( $\omega t$ ), estimada por el PLL. La ecuación (2) muestra una ecuación fasorial del perfil de potencia (S) con el voltaje (V) y la corriente (I), empleando relaciones de fase. El ángulo de la tensión es cero para establecer la referencia del sistema. La ecuación (3) permite inferir que el ángulo de la corriente $(\theta)$ es el negativo del ángulo de potencia aparente $\left(\phi_{s}\right)$. La ecuación (4) es la definición de potencia aparente para sistemas trifásicos balanceados en función de valores RMS del voltaje (VII) y corriente (iil), ambos línea a línea. Considerando la conexión en estrella y el valor pico de las señales se define la ecuación (5), relacionando la magnitud de la corriente $\left(\mathrm{I}_{\mathrm{m}}\right)$ con la potencia aparente RMS trifásica balanceada (SRMs) y la magnitud de la tensión de fase $\left(V_{m}\right)$.

$$
\begin{aligned}
& \frac{\mathrm{S} \mid \phi_{\mathrm{S}}}{\mathrm{V}[0}=\mathrm{Il}-\theta \\
& \theta=-\phi_{\mathrm{S}} \\
& \mathrm{S}_{\mathrm{RMS}}=\sqrt{3} \mathrm{~V}_{\|} \mathrm{i}_{\mathrm{i}} \\
& \mathrm{I}_{\mathrm{m}}=\frac{2 \mathrm{~S}_{\mathrm{RMS}}}{3 \mathrm{~V}_{\mathrm{m}}}
\end{aligned}
$$

EI LP de impedancia constante se puede expresar como un LP de corriente en función de la tensión de la red de alimentación, similarmente a lo deducido con el LP de potencia constante. La ecuación (3) es la relación fasorial de la impedancia (Z) con el voltaje (V) y corriente de fase (I), con sus ángulos asociados. La ecuación (7) describe que el ángulo negativo de la impedancia $\left(\phi_{z}\right)$ es equivalente al ángulo de la corriente $(\theta)$. Además, la relación de las magnitudes de fase del voltaje $\left(V_{m}\right)$ y la corriente $\left(I_{m}\right)$ establecen la magnitud de la impedancia $\left(Z_{m}\right)$. Concluyendo, es posible considerar los perfiles de impedancia y potencia como perfiles de corriente en función de la tensión. Estas relaciones son válidas para estado estable, ya que sus deducciones son hechas a partir de ecuaciones fasoriales.

$\frac{\mathrm{V}\lfloor 0}{Z\left[\phi_{\mathrm{z}}\right.}=1 \mid \theta$

$\theta=-\phi_{z}$

$\mathrm{I}_{\mathrm{m}}=\frac{\mathrm{V}_{\mathrm{m}}}{Z_{\mathrm{m}}}$

\section{Lazo de seguimiento de fase (PLL)}

El PLL es un lazo de control oscilatorio que se encarga de acoplar dos señales, en este caso (Vu) y (Vsync). Este sistema de control está encargado de sincronizar la tensión de la red con las corrientes de referencia usando un lazo cerrado de control, por lo que la señal de salida (wt) es usada en la etapa de generación de referencias. El diagrama de control de la Figura 3 ilustra el controlador implementado. La señal medida (Va) se divide por la amplitud del armónico fundamental calculada mediante la FFT. Al dividir la señal (Va) por la amplitud, se obtiene una señal coseno de amplitud unitaria $(\mathrm{Vu})$; posteriormente, en el lazo retroalimentado se multiplica por la señal cosenoidal de la salida (Scos) y luego se ingresa al controlador (PI), a través de un filtro de segundo orden (2-F). Los parámetros del controlador se basan en la documentación de PSIM, conservando la frecuencia de corte y fase definidos en Karimi-Ghartema, (2020), ganancia de 50 y la constante de tiempo 5 [ms]. La señal triangular (PLLport) es la referencia que varía de 0 a 360 grados de forma periódica, basado en el periodo de una función coseno. La salida del controlador (PI1) suma un término al argumento para acoplar la señal. La señal que se usará principalmente es el argumento del coseno (wt) de la señal acoplada. 
Para garantizar la correcta operación de la PEL es necesario que el error de acople del PLL sea bajo, por esto se ha implementado un sistema de validación del PLL ilustrado en el recuadro superior de la Figura 3. El lazo de control del PLL entrega la señal de error, denominada (Err1). Está señal es comparada con el valor de 0.1, produciendo la señal PLLok1, que es una señal booleana que se activa cuando el Err1 es menor que 0.1 . El bloque (cont) es encargado de incrementar un contador cada milisegundo, reflejando su resultado en la señal PLLok2. Finalmente, La señal PLLok3 es booleana y se activa cuando el conteo es mayor a 17 milisegundos, relacionando el periodo de una señal de $60 \mathrm{~Hz}$. El funcionamiento de la PEL es posible solamente cuando la señal PLLok3 esté activada, con esto se busca evitar el malfuncionamiento de la PEL durante la inicialización o ante un desenganche del PLL.

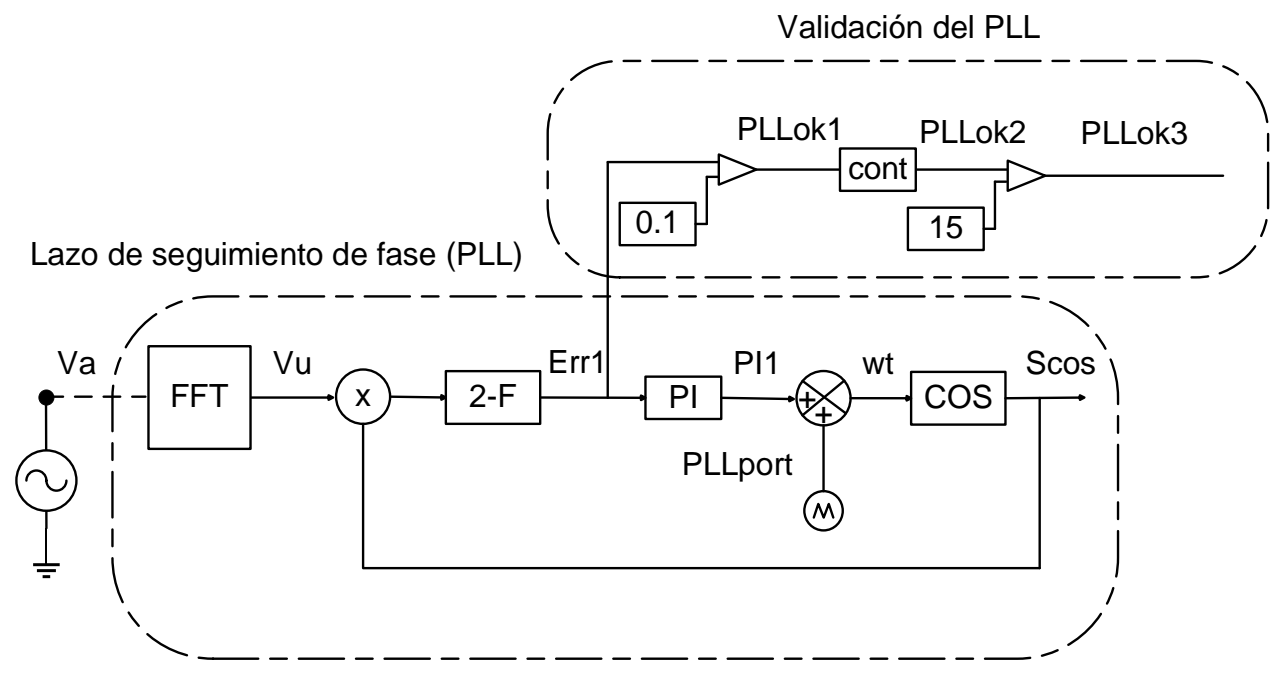

Fig. 3: Esquema de control del PLL

\section{Control de corriente por histéresis (HCC)}

El HCC es conocido por su implementación simple y robusta frente a perturbaciones y variaciones de parámetros, que le permite alcanzar condiciones de trabajo extremas (Fereidouni et al., 2016; Wang et al., 2019). La Figura 4 describe el diagrama de control del HCC, el cual tiene tres señales de entrada: la corriente de referencia (RefA), la medición de corriente (ia) y el ancho de banda $(\beta / 2)$.

Control de corriente por histéresis (HCC)

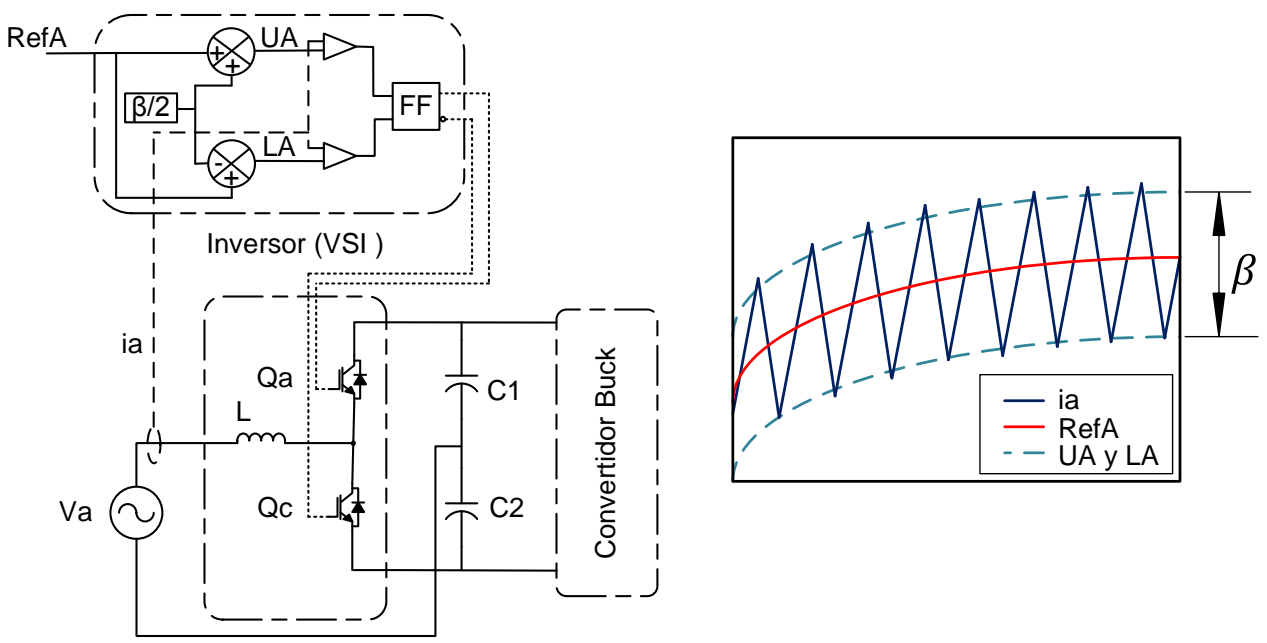

Fig. 4: Esquema de control HCC de una rama

Los límites superior (UA) e inferior (LA) de la banda de histéresis son definidos al sumar y restar $(\beta / 2)$ a la corriente de referencia (RefA). Posteriormente, los comparadores validan la corriente medida (ia) con los límites para determinar cuando la corriente (ia) se encuentra dentro de la banda de histéresis. La señal de los comparadores es llevada a un bloque de flip-flop set-reset, el cual se encarga de almacenar el ultimo estado de la comparación. Si la corriente está en aumento, el flip-flop se encarga de conservar este estado hasta que supere el límite superior y a partir de allí accionar los interruptores (Qa) y (Qc) para que la corriente (ia) empiece a disminuir su valor. Esta estrategia de control fue extrapolada a las fases b y c para completar el sistema trifásico. En Malesani y Tenti, (1990), se definen las ecuaciones (9) y (10) que representan los estados de 
conmutación del circuito de potencia (Figura 4). La tensión en cada capacitor equivale a la mitad de la tensión (E) mientras que la inductancia es representada por la letra L. Finalmente, el voltaje y corriente de la rama A, son representados por $i_{a}$ y $v_{a}$, respectivamente.

$$
\begin{aligned}
& \frac{E}{2}=L \frac{d i_{a}}{d t}+v_{a} \\
& -\frac{E}{2}=L \frac{d i_{a}}{d t}+v_{a}
\end{aligned}
$$

El tiempo de incremento $\left(t_{p}\right)$ y el tiempo de decremento $\left(t_{n}\right)$ de la corriente están dados por las ecuaciones (12) y (13) (Malesani y Tenti, 1990). Donde la variable $\left(u^{*}\right)$ es la tensión de referencia de fase, determinada por la tensión del bus DC, el desfase del inductor y la corriente deseada. El termino $\left(u^{\circ}\right)$ es la tensión de fase normalizada, en función del voltaje de referencia de fase y la tensión del bus DC. En términos generales, las ecuaciones (12) y (13) reflejan que la estrategia de control HCC depende del valor de inductancia, el ancho de banda de histéresis y la diferencia entre las tensiones de la red y el bus DC. Por otro lado, la implementación de este dispositivo se puede ver afectada al considerar la frecuencia de conmutación y muestreo fija, puesto que la estrategia no considera la digitalización de las señales y la acción de control se basa únicamente en la medición de la corriente.

$$
\begin{aligned}
& u^{\circ}=\frac{u^{*}}{(E / 2)} \\
& t_{p}=\frac{2 \beta L}{E\left(1-u^{\circ}\right)} \\
& t_{n}=\frac{2 \beta L}{E\left(1+u^{\circ}\right)}
\end{aligned}
$$

\section{Convertidor Buck}

El convertidor Buck se encarga de disipar los excesos de potencia del bus DC de la PEL. Para entender la función del Buck se debe resaltar que la etapa del inversor emula los LPs deseados, aportando energía al bus DC. Las componentes de potencia activa de los LPs son unidireccionales, cargando el bus DC y aumentando su nivel de tensión. El valor del bus DC está relacionado con las ecuaciones (12) y (13); por ende, el voltaje del bus DC está conexo con el tiempo que la corriente va a incrementar/decrementar entre los límites de la banda de histéresis, también con el límite de aislamiento eléctrico de los condensadores y los elementos asociados. Por lo tanto, definir un adecuado nivel de tensión es un balance entre velocidad de operación y soportabilidad eléctrica de los equipos. En términos generales, la etapa del inversor inyecta energía al bus DC con las componentes de potencia activa y el convertidor Buck se encarga de disipar los excesos de energía para mantener el bus DC estable.

La ecuación (14) es la función de transferencia que modela el convertidor Buck en el dominio de la frecuencia, para este modelo fue considerado el modelo de pequeña señal. La función de transferencia corresponde a el cociente entre la salida y la entrada. La salida del sistema es la tensión en el capacitor de entrada ( $v_{\text {cin }}$ ) y la entrada del sistema es la variable $(\mu)$ que representa el ciclo de trabajo de la modulación PWM (Pulse-Width Modulation) aplicada al interruptor de potencia (Qb). La etapa de Inversor (VSI) es modelada como una fuente de corriente que inyecta/extrae energía al bus DC y los capacitores C1 y C2 son agrupados en un capacitor denominado $\left(c_{i n}\right)$. Los valores denotados con la barra superior son valores operativos promedio. El modelo propuesto permite determinar el valor de los controladores del convertidor Buck.

$\frac{v_{C i n}}{\mu}=\frac{-s^{2} \bar{L}_{L b} R_{b} L_{b} C_{b}-s \bar{\mu} R_{b} C_{b} \overline{V_{C i n}}-s \overline{L b} L_{b}-R_{b} \bar{T}_{L}-\bar{\mu} \overline{V_{C i n}}}{s^{3} R_{b} L_{b} C_{i n} C_{b}+s^{2} C_{i n} L_{b}+s C_{i n} R+s C_{b} R_{b} \bar{\mu}^{2}+\bar{\mu}^{2}}$

El objetivo de este controlador es mantener un bus DC estable, el valor deseado (SP, Set Point). La Figura 5 describe el controlador implementado y el esquema de potencia, la salida del controlador PI es acondicionada por un retenedor de orden cero $(\mathrm{ZOH}$, Zero-Order Hold) que se encarga de simular la digitalización; es decir, que la referencia del PWM solo cambia con relación a la frecuencia de conmutación del sistema de control. La salida del $(\mathrm{ZOH})$ es comparada con una referencia portadora triangular construyendo así un PWM para activar el interruptor de potencia $(\mathrm{Qb})$. La potencia que extrae el convertidor Buck aumenta proporcionalmente al ciclo de trabajo del interruptor $(\mathrm{Qb})$, por lo tanto, a medida que la diferencia entre la tensión deseada y la tensión medida disminuye entonces la potencia extraída del bus DC debe disminuir para propiciar un voltaje estable, es por esto que se utiliza el bloque inversor (-1) en el esquema de control. 


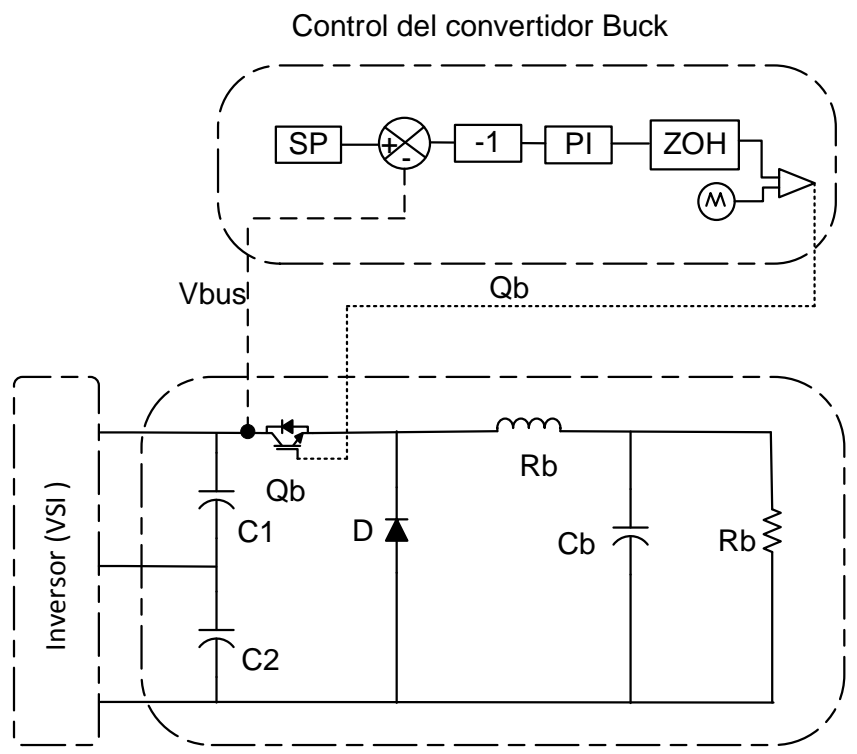

Fig. 5: Esquema de control y potencia del convertidor Buck

\section{RESULTADOS Y DISCUSIÓN}

La topología de la Figura 1 junto con su estrategia de control fueron implementadas mediante simulación. La Tabla 1 muestra los parámetros usados, el parámetro de (RI) es la resistencia serie del inductor (L) que representa sus pérdidas y $(\mathrm{Rc})$ son los resistores de estabilización que se ubican en paralelo a los capacitores (C1) y (C2). El software de simulación empleado fue PSIM, paquete orientado a la simulación de circuitos de electrónica de potencia. A continuación, se describirán los resultados de las simulaciones realizadas.

Tabla 1: Parámetros de simulación

\begin{tabular}{|l|c|c|}
\hline \multicolumn{1}{|c|}{ Parámetro } & Valor & Unidad \\
\hline Inductor de entrada (L) & 3.00 & $\mathrm{mH}$ \\
\hline Resistor serie del inductor (RI) & 0.20 & $\Omega$ \\
\hline Capacitores (C1, C2 y Cb) & 3.30 & $\mathrm{mF}$ \\
\hline Parámetro P del lazo de control del Buck & 3.73 & - \\
\hline Parámetro I del lazo de control del Buck & 7.57 & $\mathrm{~ms}$ \\
\hline Ancho de banda de HCC ( $\beta / 2)$ & 0.20 & $\mathrm{~A}$ \\
\hline Resistor del Buck (Rb) & 6.00 & $\Omega$ \\
\hline Frecuencia de conmutación & 20.00 & $\mathrm{kHz}$ \\
\hline Inductor del Buck (Lb) & 23.00 & $\mathrm{mH}$ \\
\hline Vab (línea a línea RMS) a 60Hz & 208.00 & $\mathrm{~V}$ \\
\hline Tensión del bus DC deseado & 600.00 & $\mathrm{~V}$ \\
\hline Resistor de estabilización (Rc) & 100.00 & $\mathrm{k} \Omega$ \\
\hline
\end{tabular}

Lazo de seguimiento de fase (PLL)

EI PLL es el lazo de control que se encarga de acoplar la señal de referencia $(\mathrm{Vu})$ que proviene de la red eléctrica (línea de color azul) con la señal (Vsync) que corresponde a la señal que se desea acoplar (línea de color verde), ver Figura 6.a. La Figura 6.b. contiene una señal triangular (PLLport) que es la señal portadora, esta señal va cambiando desde $0^{\circ}$ hasta $360^{\circ}$ y dura el periodo completo de la señal Vu (16.6 $\mathrm{ms}$ ) para una frecuencia de $60 \mathrm{~Hz}$. El termino (PI1) en color azul corresponde a la salida del controlador PI que debe sumarse a la señal PLLport. La señal wt es la suma de PLLport y PI1, por lo tanto, wt contiene el argumento de la señal Vu, este argumento se utiliza como entrada en el sistema "Generación de referencias".

La Figura 6 fue obtenida simulando la etapa de PLL sin considerar la FFT para facilitar la comprensión de la operación del PLL, ya que la FFT tarda alrededor de dos ciclos de señal en entregar un valor estable, perturbando el PLL. En contraposición, la Figura 7 considera la implementación de la FFT, ya que representa los principios de operación de una forma más cercana a la implementación del equipo. La Figura 7.a. ilustra las corrientes emuladas por la PEL, note que las corrientes generadas en un tiempo menor a 0.1 segundos no son señales sinusoidales de un sistema trifásico. Consecuentemente, el diseño de la estrategia de control establece una etapa de control que se encarga de validar el acople de las señales por parte del PLL. A continuación, es presentado el esquema de control empleado y su resultado se puede observar en la Figura 
7.b. Note que las corrientes emuladas por la PEL son sinusoidales y balanceadas, ya que hubo una validación previa del PLL. La emulación de corrientes es nula previa a 0.1 segundos para asegurar un adecuado funcionamiento. La Figura 8. ilustra la operación del esquema de control que garantiza que la señal Err1 sea menor a 0.1 por 17 milisegundos para habilitar la emulación de corrientes de la PEL.
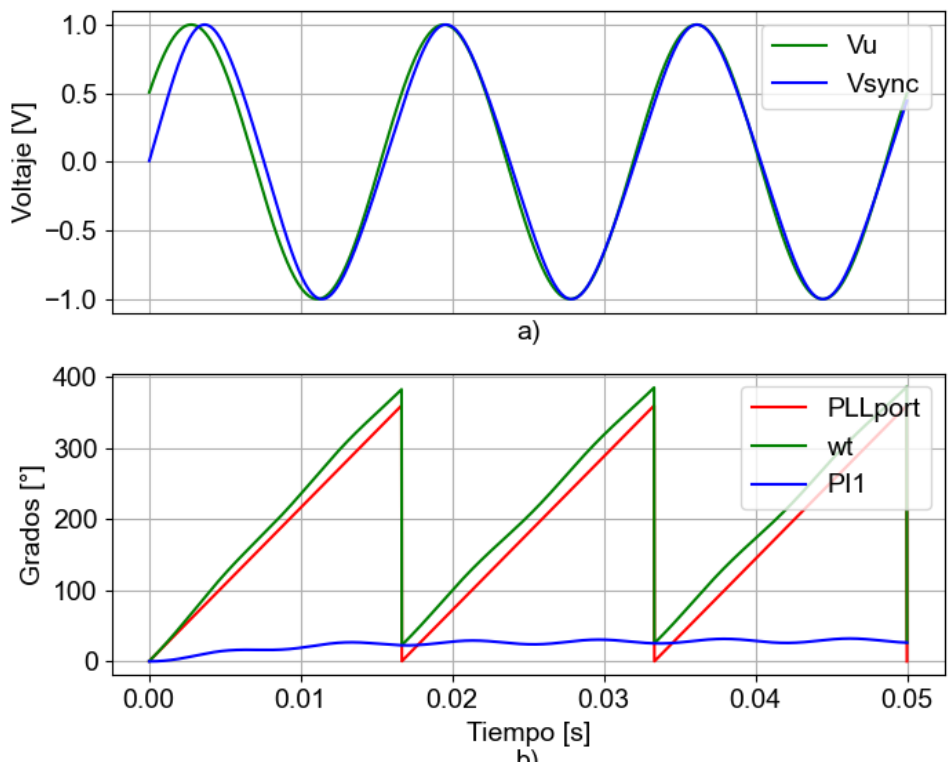

Fig. 6: Acople de señales mediante el PLL

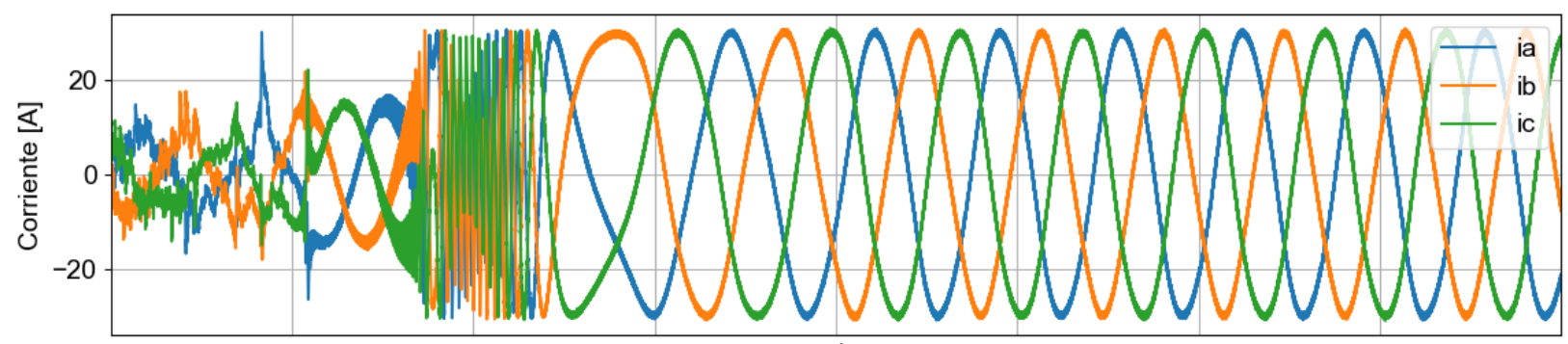

a)

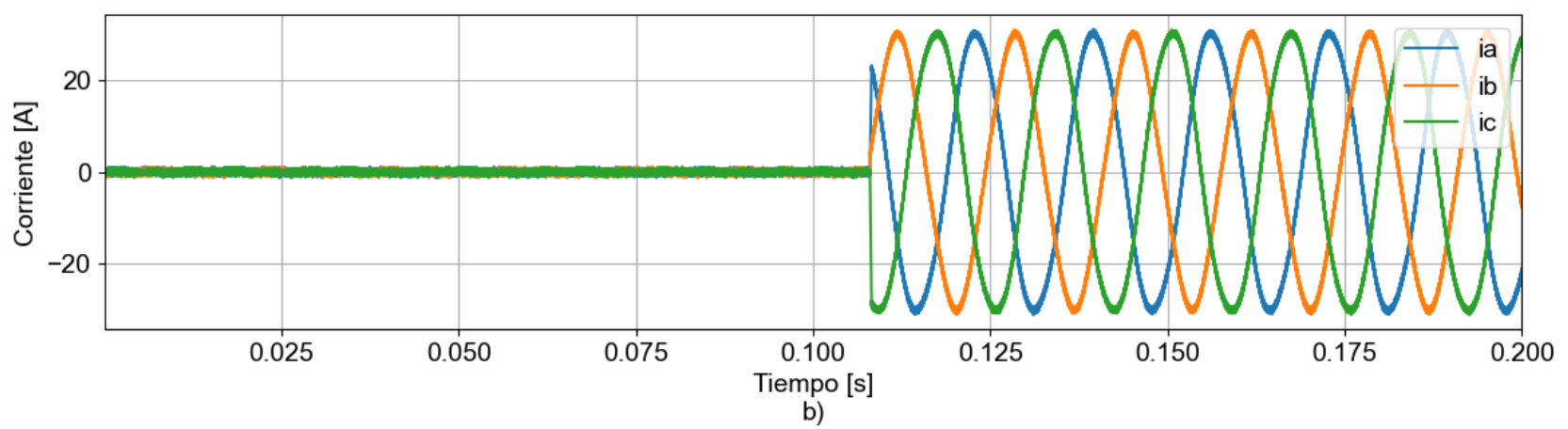

Fig. 7: Curvas de control del establecimiento del PLL

La Figura 8.a. ilustra la señal de referencia unitaria $(\mathrm{Vu})$ que proviene de la red eléctrica y la señal (Vsync) que corresponde a la señal que se desea acoplar, observe que al iniciar la señal Vsync no coincide con la señal de referencia $(\mathrm{Vu})$ pero el lazo de control del PLL se encarga de modificar el argumento ( $\omega t)$ para acoplar las señales sinusoidales. La Figura 8.b. muestra el comportamiento de la señal Err1, también se muestra la banda de 0.1 , evidenciando un valor tolerable. Cabe mencionar que el PLL es un controlador oscilatorio, por lo cual se observa un comportamiento sinusoidal del error. La Figura 8.c. es la señal booleana PLLok1 que se activa cuando la señal Err1 en valor absoluto es menor a 0.1. La Figura 8.d. describe la señal PLLok2 que es la salida del contador (cont) que incrementa una unidad cada milisegundo hasta alcanzar los 17 ms. Finalmente, la Figura 8.e. ilustra la señal booleana PLLok3 que se activa cuando la señal PLLok2 es superior a 15. 

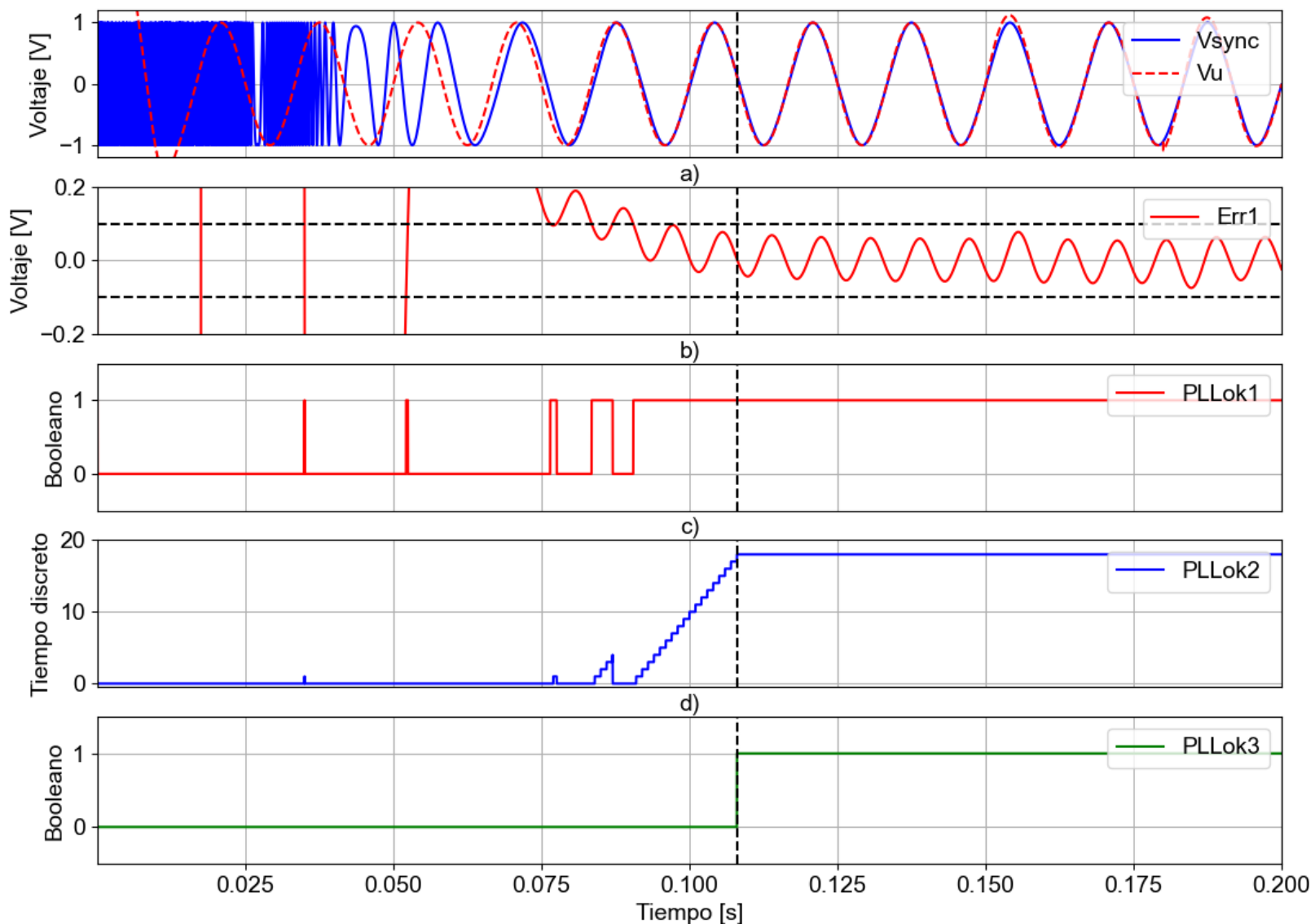

e)

Fig. 8: Validación de acople del PLL

Control de corriente por histéresis (HCC)

Esta sección describe el control HCC implementado en el VSI, usando el modo de corriente constante de magnitud 30 amperios pico y fase de 45 grados en atraso. La Figura 9 contiene las corrientes de salida del inversor. La Figura 9.a muestra las corrientes de las fases a, b y c; mientras que la Figura 9.b corresponde a la ampliación del recuadro rojo de la Figura 9.a. En la Figura 9.b., pueden verse: la corriente de referencia (RefA), la corriente de salida del inversor de la fase a (ia) y la banda superior e inferior denominadas (UA) y (LA), respectivamente, definiendo la banda de histéresis.
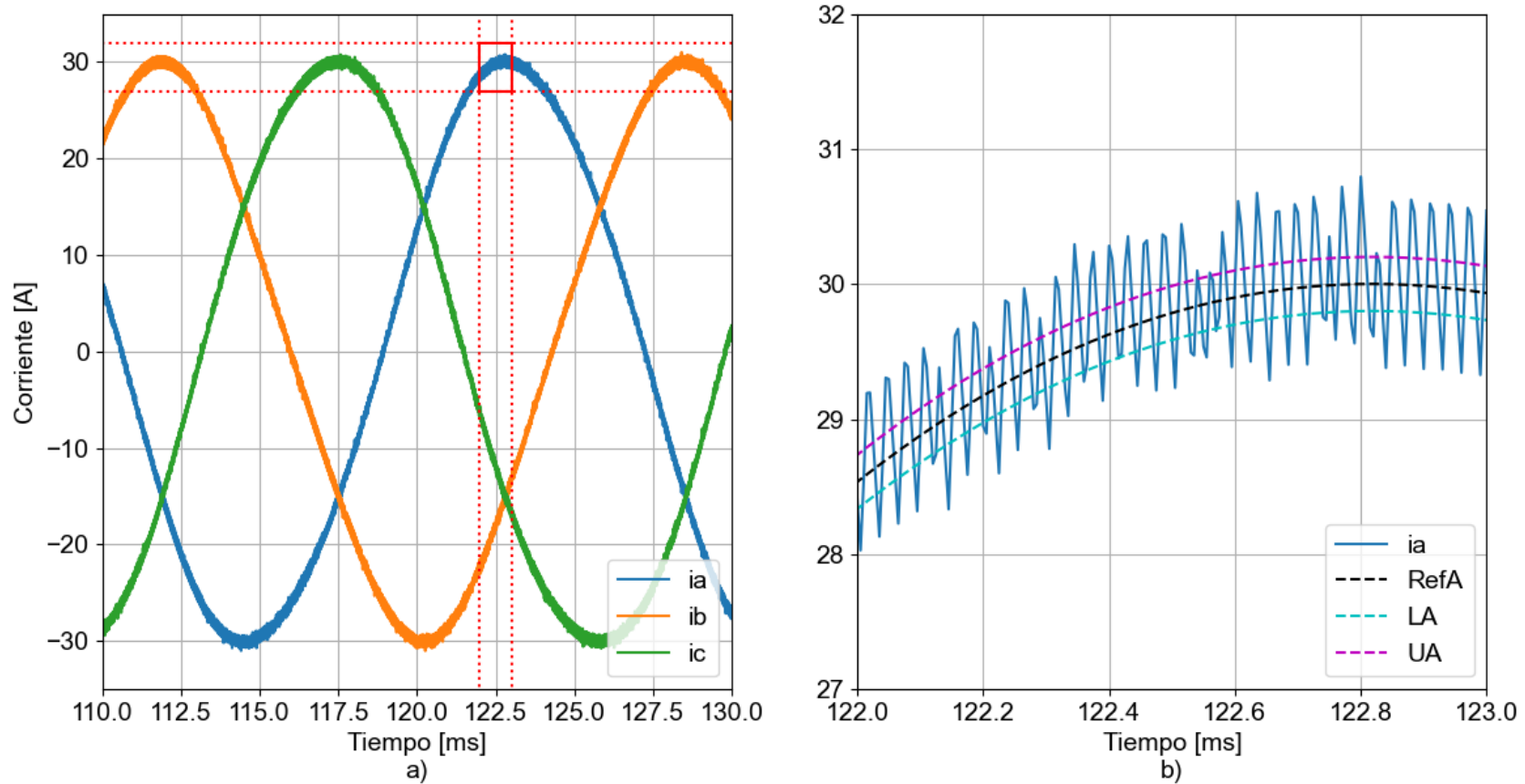

Fig. 9: Curvas de referencia y corriente del HCC 


\section{Funcionamiento sistemático de la PEL}

La Figura 10 contiene cuatro columnas que describen el funcionamiento de la PEL. La primera columna Rama $A$ muestra el voltaje de la red (Va) y la corriente emulada por la PEL (ia) de la fase A. La segunda columna Potencia $A C$ se basa en el producto de tensiones y corrientes de cada rama del sistema trifásico, calculando las potencias aritméticas instantáneas de cada una ( $\mathrm{Sa}, \mathrm{Sb}$ y Sc) y la suma de ellas (S3) en unidades de kVA. La tercera columna Voltaje bus DC describe la tensión en el bus DC (VbusDC) y la referencia del bus (RefBUS), parametrizado en 600 [V]. La cuarta columna Potencia $D C$ describe la potencia en el resistor Rb (PR). Note que las dos primeras columnas tienen una escala temporal de 20 milisegundos, iniciando en 135 [ms], debido al tiempo inicial que tarda el PLL en habilitar la generación de referencias. Mientras que, las dos últimas columnas tienen escala de 200 [ms]. Estas escalas temporales han sido empleadas debido a la observabilidad del fenómeno, por ejemplo, en la primera columna son ilustradas señales periódicas, por ende, un periodo de la señal describe el fenómeno estudiado. Las filas de la Figura10 representan escenarios operativos de carga, la fila (A) representa un LP inductivo puro, la fila (B) un LP inductivo-resistivo y la fila (C) un LP resistivo puro.

La Figura 10 representa las principales variables de funcionamiento de la PEL. A continuación, las repuestas son analizadas por columna comparando los resultados en cada escenario operativo: 1) Rama A: la magnitud de corriente de 30 amperios pico y ángulos de $-90,-45$ y 0 grados, para los escenarios A, B y C respectivamente, son visualizados en la columna. 2) Potencia $A C$ : la potencia reactiva genera flujos bidireccionales, estableciendo una potencia trifásica (S3) media igual a cero, como se observa en el escenario A. La potencia activa genera flujos unidireccionales, evidenciando en el escenario C (S3). El LP inducto-resistivo en el escenario B ilustra la fluctuación de la potencia reactiva y la componente DC de la potencia trifásica (S3), producido por la potencia activa. 3) Voltaje bus $D C$ : el objetivo del convertidor Buck es retirar los excesos de potencia para mantener el voltaje del bus DC constante, sin embargo, en el LP inductivo puro (escenario A) la potencia media es igual cero, por lo tanto, no hay excesos de energía para el bus, pero las pérdidas de conmutación y conducción consumen energía lo que produce la descarga del bus, como se visualiza en la gráfica del Voltaje del bus DC del escenario A. Consecuentemente, el escenario A permite inferir que la topología de la PEL simulada requiere un consumo mínimo de potencia activa para compensar las pérdidas. Por el contrario, los escenarios B y C inyectan energía en el bus DC, entonces el convertidor Buck estabiliza el bus $\mathrm{DC}$ en el valor deseado de 600 voltios.

Note la perturbación ilustrada al inicio de la gráfica, producida al iniciar la emulación de los LPs. 4) Potencia $D C$ : $\mathrm{La}$ acción del convertidor Buck se refleja en la potencia consumida en el resistor (Rb), reflejando la potencia extraída al bus DC. EI LP inductivo puro no entrega excesos de potencia, por lo que el convertidor Buck no extrae potencia, para evitar que el bus DC se descargue más rápidamente. Los escenarios $\mathrm{B}$ y $\mathrm{C}$ extraen apropiadamente corriente del bus DC siendo mayor la cantidad de C por tener una componente de potencia activa mayor a B. Note la relación en magnitud entre la potencia en AC y DC. Finalmente, las gráficas permiten concluir que la simulación de la PEL funciona adecuadamente como sistema, respondiendo a diferentes escenarios operativos y conservando la estabilidad de la red.
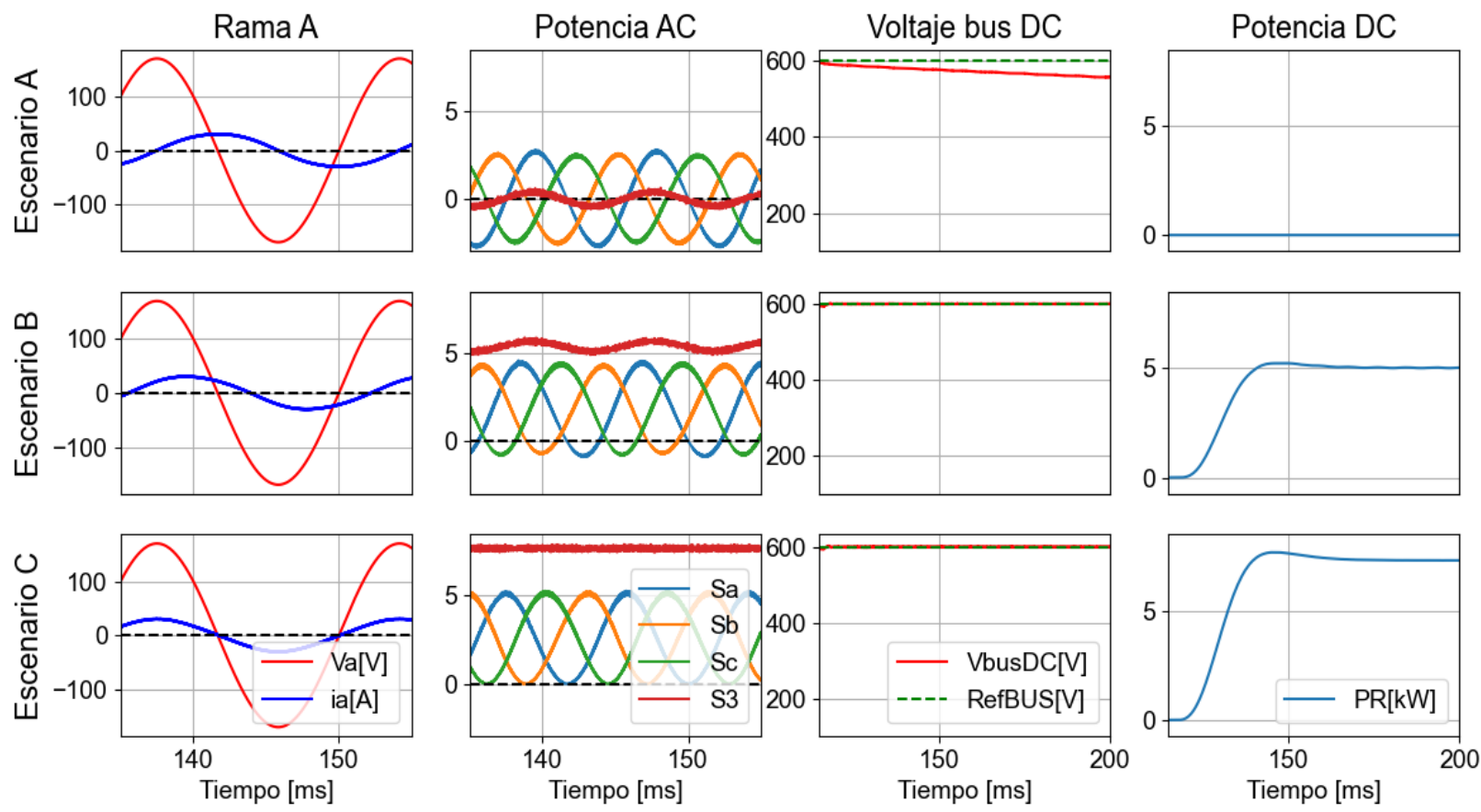

Fig. 10: Escenarios de la PEL, formas de onda 


\section{Modos de operación de la PEL}

La simulación desarrollada emula diferentes LPs según la parametrización del usuario, por lo que, la PEL responde a la alimentación del sistema en función al modo de operación establecido. La PEL fue alimentada con tensiones variables para nueve escalones de tiempo. La Tabla 2 ilustra los valores RMS de tensión línea a línea (vll) que fueron establecidos en la fuente de alimentación trifásica con conexión en estrella, paralelamente el tiempo de los escalones (T) en los cuales fueron aplicados los cambios de tensión. La Figura 11.a. describe las tensiones de fase de cada rama y la línea discontinua de color negro refleja el tiempo de cada escalón. Las filas LP S, LP Z y LP I representan los perfiles de potencia, impedancia y corriente constante. El LP de potencia constante (Figura 12.a) fue parametrizado con 10806 [VA] de potencia aparente aritmética trifásica y factor de potencia de 0.707 en atraso. EI LP de impedancia constante fue parametrizado con 4 ohmios de magnitud pico y ángulo de 45 grados. Finalmente, El LP de corriente constante (Figura12.c) fue parametrizado para una magnitud de $42.42[\mathrm{~A}]$ y ángulo de 45 grados en atraso.

Tabla 2: Escalones de tensión para probar los modos de operación de la PEL

\begin{tabular}{|c|c|}
\hline $\mathrm{T}[\mathrm{s}]$ & $\mathrm{vll}[\mathrm{v}]$ \\
\hline$<0.15$ & 124.8 \\
\hline 0.15 & 145.6 \\
\hline 0.18 & 166.4 \\
\hline 0.21 & 187.2 \\
\hline 0.24 & 208.0 \\
\hline 0.27 & 228.8 \\
\hline 0.30 & 249.6 \\
\hline 0.33 & 270.4 \\
\hline 0.36 & 291.4 \\
\hline
\end{tabular}

Las figuras 10.b., 10.c. y 10.d. son las corrientes de la fase $A$, cambiando la magnitud en función del perfil parametrizado. Las filas LP S, LP Z y LP I representan los perfiles de potencia, impedancia y corriente constante, razón por la cual el perfil de impedancia constante (LP Z) aumenta la corriente proporcionalmente al aumento de tensión; el perfil de potencia constante (LP S) ilustra una relación inversamente proporcional entre el voltaje y la corriente. Finalmente, el perfil de carga de corriente constante (LP I) muestra la corriente invariante a pesar de los cambios en tensión de la fuente trifásica de alimentación. La validación de los modos de operación se observa en la Figura 12, demostrando las relaciones de tensión y corriente según el perfil de carga parametrizado. La Figura 12 fue construida con la magnitud de corriente y tensión medidas en simulación, posteriormente se relacionan sus valores por cada perfil, validando la tendencia asociada de cada modo de operación.
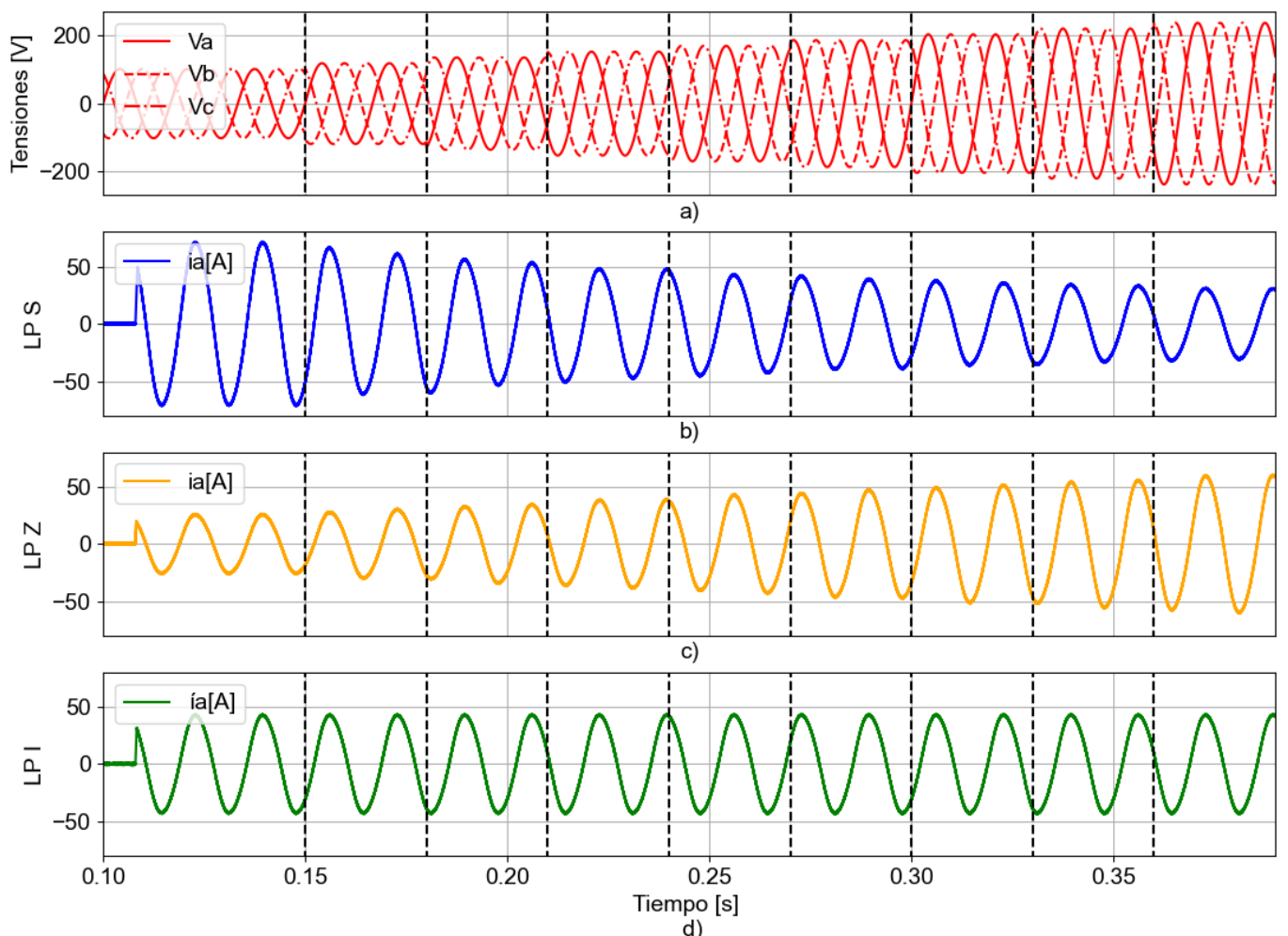

Fig. 11: Modos de operación de la PEL 


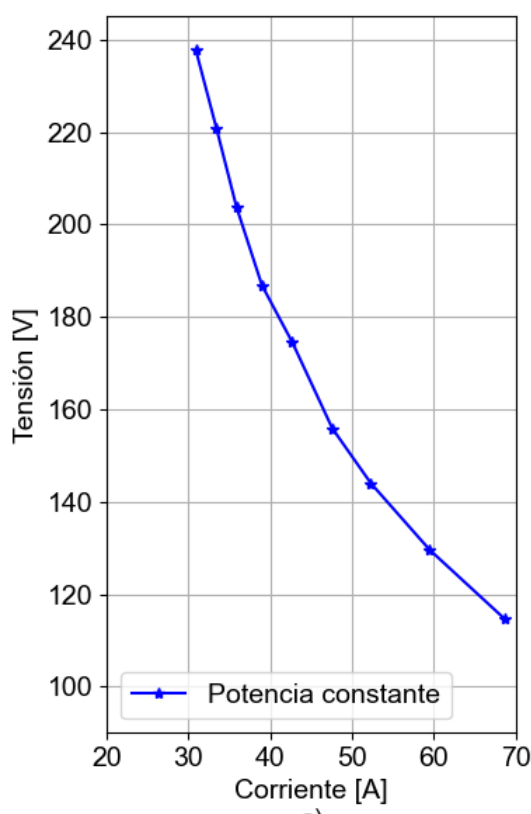

a)

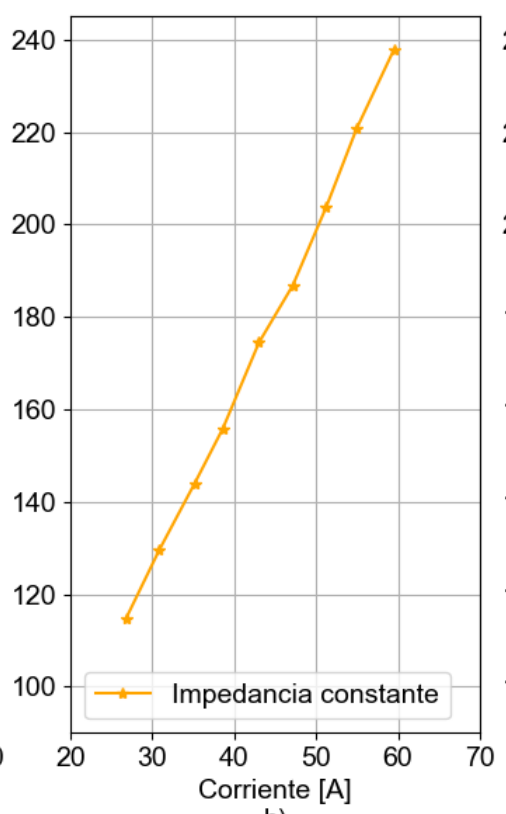

b)

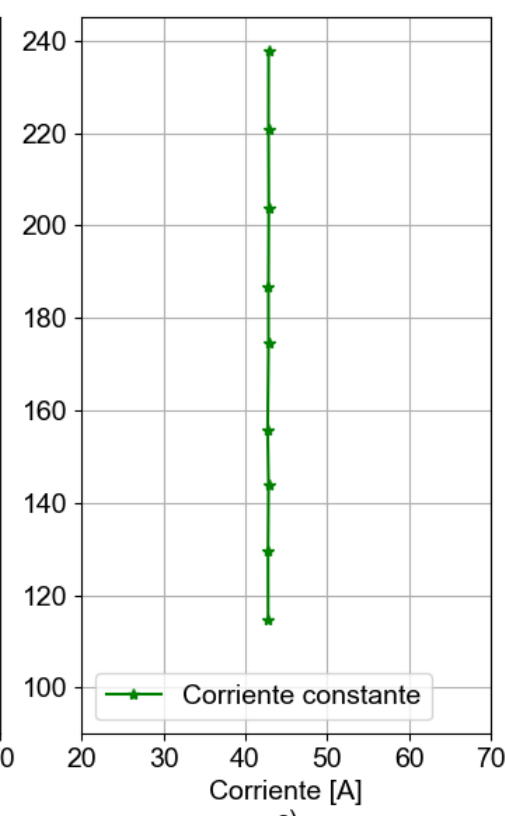

c)

Fig. 12: Validación de los perfiles de carga

\section{CONCLUSIONES}

A partir de los resultados obtenidos, se pueden extraer las siguientes conclusiones: 1) La simulación de la PEL funciona de manera adecuada, representando las formas de onda de la etapa de potencia y las señales de control de acuerdo con la teoría. 2) EI PLL implementado acopla las señales de alimentación de la red y calcula el ángulo de referencia que permita la generación de referencias trifásicas. El PLL implementado estima la frecuencia angular en un tiempo inferior a $125 \mathrm{~ms}$, asegurando condiciones iniciales seguras para la operación de la PEL. 3) El control del PLL garantiza el acople entre señales para operar la PEL sin tener riesgos de generar señales deformadas que no correspondan con los modos de operación establecidos. 4) El HCC implementado opera de forma adecuada, la banda de histéresis y su control asociado son eficaces para seguir la referencia de corriente definida. Las curvas obtenidas ilustran la corriente emulada por la PEL, siguiendo la banda de histéresis propuesta en el control. 5) El funcionamiento de la PEL como sistema es adecuado, ya que la operación de la PEL emula diferentes escenarios de carga, también conserva la tensión en el bus DC dentro de su rango operativo. Las gráficas permiten concluir que el perfil inductivo puro no se puede emular por un tiempo prolongado, debido al consumo de energía de las pérdidas de conducción y conmutación que disminuyen la tensión del bus DC, estableciendo así una restricción operativa. 6) La PEL fue diseñada para operar en los modos de potencia, impedancia y corriente constante; los resultados mostrados en el presente artículo evidencian la representación adecuada de los tres modos de operación en función de la tensión de alimentación. Los modos operativos de la PEL fueron obtenidos con las ecuaciones deducidas en el presente artículo, comprobando que los perfiles de potencia e impedancia constante pueden ser procesados como un perfil de corriente dependiente de la tensión de alimentación, conservando la relación tensión-corriente de cada modo de operación. 7) El convertidor Buck es encargado de disipar los excesos de potencia para mantener estable la tensión del bus DC. En el presente artículo se evidencia que los parámetros del controlador PI del convertidor Buck reflejan un desempeño estable frente a las perturbaciones sometidas de la PEL. En términos generales, el control HCC PI implementado en la PEL responde de forma satisfactoria ante los diferentes escenarios simulados, ya que hay una adecuada emulación de perfiles de carga, ya sean corriente, impedancia o potencia constante. Los controladores del PLL y el convertidor Buck han sido sintonizados eficazmente para contribuir a la estabilidad sistémica del dispositivo.

\section{REFERENCIAS}

Arias, L., Rivas, E., y León, L., Propuesta de modelo de Gestión para redes eléctricas con Generación Distribuida a través de Unidades de Medición Fasorial, http://dx.doi.org/10.4067/S0718-07642017000200003, Info. Tecnol., 28(2), $15-28$ (2017).

Aurilio, G., Gallo, D., Landi, C., y Luiso, M., AC Electronic Load for On-Site Calibration of Energy Meters, https://doi.org/10.1109/I2MTC.2013.6555519, 2013 IEEE International Instrumentation and Measurement Technology Conference (I2MTC), 768-773 (2013).

Fereidouni, A., Masoum, M. A. S. y Smedley, K. M., Supervisory Nearly Constant Frequency Hysteresis Current Control for Active Power Filter Applications in Stationary Reference Frame, https://doi.org/10.1109/JPETS.2015.2501423, IEEE Power and Energy Technology Systems Journal, 3(1), 1-12 (2016). 
Geng, Z., Gu, D., Hong, T., y Czarkowski, D., Programmable Electronic AC Load Based on a Hybrid Multilevel Voltage Source Inverter, https://doi.org/10.1109/TIA.2018.2818059, IEEE Transactions on Industry Applications, 54(5), 55125522. (2018).

Gómez, V. A., Hernández, C., y Rivas, E., La Influencia de los Niveles de Penetración de la Generación Distribuida en los Mercados Energéticos, http://dx.doi.org/10.4067/S0718-07642018000100117, Info. Tecnol., 29 (1), 117-128 (2018).

Kapat, S., Parameter-Insensitive Mixed-Signal Hysteresis-Band Current Control for Point-of-Load Converters With Fixed Frequency and Robust Stability, https://doi.org/10.1109/TPEL.2016.2608913, IEEE Transactions on Power Electronics, 32(7), 5760-5770 (2017).

Karimi-Ghartema, M., Tutorial-Implementation and Design of PLL and Enhanced PLL Blocks [Support PSIM], Implementation and Design of PLL and Enhanced PLL Blocks, (2020).

Kim, K., Lee, H., Hong, S., y Cho, G., A Noninverting Buck-Boost Converter With State-Based Current Control for Li-ion Battery Management in Mobile Applications, https://doi.org/10.1109/TIE.2018.2883257, IEEE Transactions on Industrial Electronics, 66(12), 9623-9627 (2019).

Malesani, L., y Tenti, P., A Novel Hysteresis Control Method for Current-Controlled Voltage-Source PWM Inverters with Constant Modulation Frequency, https://doi.org/10.1109/28.52678, IEEE Transactions on Industry Applications, 26(1), 88-92 (1990).

Manbachi, M., y Ordonez, M., Intelligent Agent-Based Energy Management System for Islanded AC-DC Microgrids, doi: https://doi.org/10.1109/TII.2019.2945371, IEEE Transactions on Smart Grid, 16 (7), 4603-4614 (2020).

Novak, D., Beraki, M., Villa, G., y García, P., Low-Cost Programmable Three Phase Load for Microgrids Labs, https://doi.org/10.1109/EEEIC.2015.7165231, 2015 IEEE 15th International Conference on Environment and Electrical Engineering (EEEIC), 599-604 (2015).

Restrepo-Garcés, A. R., Manotas-Duque, D. F., \& Lozano, C. A., Portafolio para Auto-Generación de Electricidad con Fuentes Renovables en Edificios Comerciales, https://dx.doi.org/10.4067/S0718-07642016000100011, Info. Tecnol., 27(1), 91-104 (2016).

Serna-Montoya, L. F., Cano-Quintero, J. B., Munoz-Galeano, N., y Lopez-Lezama, J. M., Programmable Electronic AC Loads: A Review on Hardware Topologies, https://doi.org/10.1109/PEPQA.2019.8851542, 2019 IEEE Workshop on Power Electronics and Power Quality Applications (PEPQA), 1-6 (2019).

Saldarriaga-Zuluaga, S., Lopez-Lezama J.M., y Muñoz-Galeano, N., Optimal Coordination of Overcurrent Relays in Microgrids Considering a Non-Standard Characteristic, https://doi.org/10.3390/en13040922, Energies, 13(4), 922, (2020).

Srinivasa Rao, Y., y Chandorkar, M. C. Real-Time Electrical Load Emulator Using Optimal Feedback Control Technique, https://doi.org/10.1109/TIE.2009.2037657, IEEE Transactions on Industrial Electronics, 57(4), 1217-1225 (2010).

Vukojevic, A., y Lukic, S., Microgrid Protection and Control Schemes for Seamless Transition to Island and Grid Synchronization, https://doi.org/10.1109/TSG.2020.2975850, IEEE Transactions on Smart Grid, 11(4), 2845-2855 (2020).

Wang, Y., Ruan, X., Leng, Y. y Li, Y., Hysteresis Current Control for Multilevel Converter in Parallel-Form Switch-Linear Hybrid Envelope Tracking Power Supply, https://doi.org/10.1109/TPEL.2018.2835640, IEEE Transactions on Power Electronics, 34(2), 1950-1959 (2019). 
